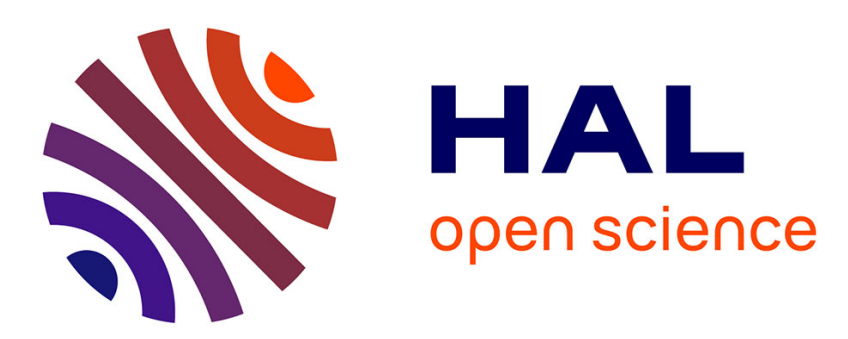

\title{
Simulations of sheared dense noncolloidal suspensions: Evaluation of the role of long-range hydrodynamics
}

\author{
Stany Gallier, François Peters, Laurent Lobry
}

\section{To cite this version:}

Stany Gallier, François Peters, Laurent Lobry. Simulations of sheared dense noncolloidal suspensions: Evaluation of the role of long-range hydrodynamics. Physical Review Fluids, 2018, 3 (4), pp.042301. 10.1103/PhysRevFluids.3.042301 . hal-01802715

\section{HAL Id: hal-01802715 https://hal.science/hal-01802715}

Submitted on 29 May 2018

HAL is a multi-disciplinary open access archive for the deposit and dissemination of scientific research documents, whether they are published or not. The documents may come from teaching and research institutions in France or abroad, or from public or private research centers.
L'archive ouverte pluridisciplinaire HAL, est destinée au dépôt et à la diffusion de documents scientifiques de niveau recherche, publiés ou non, émanant des établissements d'enseignement et de recherche français ou étrangers, des laboratoires publics ou privés. 


\title{
Simulations of sheared dense noncolloidal suspensions: Evaluation of the role of long-range hydrodynamics
}

\author{
Stany GALLIER* \\ ArianeGroup, Le Bouchet Research Center, 91710 Vert le Petit, France \\ François PETERS and Laurent LOBRY \\ Institute of Physics of Nice, UMR 7010, 06108 Nice, France
}

(Dated: February 22, 2018)

\begin{abstract}
This work intends to evaluate the role of many-body long-range hydrodynamics by simulations of sheared neutrally-buoyant non-Brownian, non-colloidal suspensions. Three-dimensional simulations of sheared suspensions are conducted with and without long-range hydrodynamics, for a volume fraction range between 0.1 to 0.62 (frictionless) and 0.1 to 0.56 (frictional). Discarding long-range hydrodynamics has only a moderate effect on viscosity for the range of volume fractions investigated and viscosities diverge with similar scaling laws ; the critical fraction is found to be approximately 0.64 (frictionless) and 0.58 (frictional). Conversely, many-body hydrodynamics are found to affect diffusion and particle velocities, which are correlated on a longer range when long-range interactions are included, even in dense suspensions. This means that long-range hydrodynamics may not be significantly screened by crowding. Assuming only short-range lubrication interactions is therefore suitable for predicting viscosity in non-colloidal suspensions but becomes questionable when flow details (e.g., diffusion or velocity correlations) are needed.
\end{abstract}




\section{INTRODUCTION}

Dense suspensions are ubiquitous in nature and industry. They exhibit an intricate physics which is far from being understood completely [1]. Numerical simulations of dense suspensions have become increasingly popular as a way to disentangle this complex physics. Particularly, simulations at the particle scale level are instrumental since they rest on fundamental principles and therefore require minimal assumptions. They have eventually fostered significant progress in current understanding, like discontinuous shear-thickening [2] for instance.

Several numerical methods are available in the literature for dilute to moderately dense suspensions. However for very dense suspensions (i.e., close to jamming), molecular dynamics (MD) is the preferred approach [2-6]. It considers particles as a system of mass points subject to repulsive forces (e.g., contact or electrostatic) and short-range hydrodynamic forces (lubrication), therefore neglecting long-range many-body hydrodynamic interactions. Other methods like Stokesian dynamics [7], force-coupling method [8] or fictitious domains [9] do incorporate long-range hydrodynamics by solving Stokes equations but at the cost of increased algorithmic complexity and computational demand, especially in dense

regimes. This explains the popularity of MD simulations for dense suspensions: they are fast and simple to develop. As far as we know, there is only one reported simulation on a suspension close to jamming which includes long-range hydrodynamics using Stokesian dynamics [10].

Long-range interactions are so exceedingly difficult to model that they are neglected in most popular simulation algorithms for suspension mechanics; this prompts the study of the regimes and scenarios in which it is valid or reasonable to make such simplifications. We are not aware of any reported studies of non-colloidal suspensions proving whether those interactions could be effectively discarded or not and if so, for which volume fraction range. This point has only recently been considered in the literature, for Brownian suspensions, to address the fact that it is generally accepted that long-range hydrodynamics are negligible in dense sheared suspensions because first, they should be rapidly screened and second, contact and divergent lubrication forces prevail.

There are very scarce works dedicated to the role of long-range hydrodynamics on rheology and, as far as we know, none in non-colloidal non-Brownian regimes. Yet, in colloidal 
suspensions, recent studies have acknowledged a notable influence of long-range hydrodynamics on the gelation and aggregation of colloids [11, 12]. Also, by studying numerically how pair mobility decays, Zia et al. [13] have showed that a long-ranged coupling persists on large distances regardless of particle concentration. This is a clear evidence that - in colloidal suspensions at least - many-body hydrodynamics are an essential part of concentrated suspension dynamics and that it should not be neglected beyond a lubrication range as usually done in MD simulations. An objective of our work is to verify if this importance of long-range interactions also persists in non-colloidal regimes.

This study therefore intends to evaluate the role of long-range hydrodynamic interactions in sheared dense non-Brownian, non-colloidal suspensions. For a wide range of volume fractions - from dilute to close-jamming - the suspension flow is computed first by solving Stokes equations (referred to as full-Stokes (FS) solution) and second, by Molecular Dynamics (MD). The comparison between FS and MD solutions will help estimate the possible role of long-range interactions. Both simulations are performed in exactly the same way (same contact or lubrication models, flow conditions, numerical parameters, etc.) so that the only difference remaining is the long-range hydrodynamics. Note that this paper only focuses on non-Brownian, non-colloidal suspensions: Brownian/colloidal interactions may result in a different physics and results obtained in this study may not be transposed straightforwardly to such regimes.

\section{METHOD}

The suspensions considered in this work are non-Brownian and composed of bidisperse spheres of radius $a_{1}$ and $a_{2}$ with $a_{2} / a_{1}=1.4$ and same volume fraction $\left(\phi_{1}=\phi_{2}\right)$ in order to avoid crystallization at high fractions. The average radius $\bar{a}$ is here defined as $\bar{a}=\left(a_{2}+a_{1}\right) / 2$. A slight polydispersity could also have been considered but bidispersity is easier to implement and this choice of parameters is widely used in the literature and reported to be quite efficient. However, this bidispersity is expected to affect rheology through a modification of the critical fraction $\phi_{m}$ at which viscosity diverges. Shauly et al. [14] have proposed an expression for the critical fraction $\phi_{m}$ in bidisperse suspensions and based on their work, we have computed the critical fraction to differ only by $2 \%$ from its monodisperse counterpart, so that limited effects of bidispersity on rheology are expected. Fluid and particle inertia 
are neglected. Full-Stokes (FS) simulations are based on a fictitious domain method which solves the Stokes flow at the particle scale and explicitly accounts for long-range many-body hydrodynamic interactions between particles [15]. A correction procedure is considered for lubrication interactions similarly to Stokesian Dynamics [7] and is more detailed in Ref. [9]. It explicitly accounts for all motions (squeezing, shearing, pumping, twisting). Let $X$ be a generic resistance function, the FS strategy then uses the correction $X^{\text {theo }}-X^{r}$ to be added as an external force/torque where $X^{\text {theo }}$ is the theoretical lubrication interaction and $X^{r}$ is that part of hydrodynamic interaction already explicitly resolved by the Stokes solver (estimated a priori). Theoretical resistance functions $X^{\text {theo }}$ depend on particle size ratio, pair orientation and normalized gap distance $\xi$. They have divergent terms (in $\xi^{-1}$ or $\ln \xi$ ) and constant $\mathcal{O}(1)$ terms. The MD simulations closely follow the approach used by [2] for lubrication and consider only the most divergent terms of $X^{\text {theo }}$ without any $\mathcal{O}(1)$ terms. In addition, a one-body Stokes drag is included in the lubrication system for all the particles [2]. Consequently, particles not interacting through short-range lubrication have Stokes drag, torque, and stresslet $S_{\infty}=10 / 3 \pi a^{3} \dot{\gamma}$ with $\dot{\gamma}$ the shear rate. This is the most widely used approach to handle lubrication although some slight variations can be found in the literature $[6,16]$. For both MD and FS, a lubrication grand resistance matrix is built in a pairwise fashion.

We consider rough particles having a reduced roughness size $h_{r} / \bar{a}=5.10^{-3}$, which is a typical experimental value [17]. Particle roughness involves actual contacts - despite lubrication - that are modeled by a normal contact force $\boldsymbol{F}^{c}$ given by a Hertz law $\boldsymbol{F}^{c}=$ $k_{n}|\delta|^{3 / 2} \cdot \boldsymbol{n}$ where $\boldsymbol{n}$ is the normal vector $\boldsymbol{n}=\boldsymbol{r} /\|\boldsymbol{r}\|$ with $\boldsymbol{r}$ the branch vector joining the sphere centers and $\delta=\|\boldsymbol{r}\|-a_{1}-a_{2}-h_{r}$. The stiffness $k_{n}$ is chosen large so as to mimic rigid particles (i.e., $|\delta| \ll \bar{a}$ ) and is controlled by the non-dimensional number $\dot{\Gamma}=6 \pi \eta \bar{a}^{2} \dot{\gamma} / k_{n} h_{r}^{3 / 2}$ (where $\dot{\gamma}$ is the shear rate and $\eta$ the fluid viscosity) which is a measure of hydrodynamic forces relative to contact forces. A small value of $\dot{\Gamma}=10^{-3}$ is prescribed in this work to obtain rigid particles. With this choice, it has been a posteriori checked that the maximum overlap $\delta$ never exceeds $h_{r}$, i.e. $\max |\delta| / \bar{a} \lesssim 5 \cdot 10^{-3}$, for all the simulations presented. Friction is accounted for by a tangential contact force $\boldsymbol{F}_{t}^{c}$ modeled by a linear spring (with tangential stiffness $\left.k_{t} / k_{n}=2|\delta|^{1 / 2} / 7\right)$ and bounded by a Coulomb-Amontons friction law with constant dynamic friction coefficient $\mu_{d}$, i.e. $\mathbf{F}_{t}^{c}=\mu_{d}\left|\mathbf{F}_{n}^{c}\right| \cdot \mathbf{F}_{t}^{c} /\left|\mathbf{F}_{t}^{c}\right|$. Once all forces are computed (contact, short-range lubrication, long-range hydrodynamics), particle positions are advanced 
using a second-order Adams-Bashforth scheme.

In MD simulations, only contact and short-range lubrication are considered to update particle positions. Long-range interactions are therefore the only difference between FS and MD simulations since all numerical parameters/algorithms/models are kept identical otherwise. An important remark however is that since MD does only have short-range lubrication interactions to model the fluid, those interactions must be truncated at some point (because theoretical lubrication functions used are only valid for very close particles). Said differently, lubrication forces are computed pairwise only among nearest neighboring particles, up to a particle gap distance $h_{\max }$. This therefore involves a free parameter, usually in the range $h_{\max } / \bar{a}=0.05 \sim 0.1$. For gaps $h>h_{\max }$, particles are supposed to behave as isolated particles with Stokes law, as mentioned previously. Note that this $h_{\max }$ cut-off only exists for molecular dynamics (MD) and not Stokes simulations. This $h_{\max }$ is different from another cut-off in Stokes solvers below which lubrication corrections are activated (lubrication correction cut-off distance). Beyond this lubrication correction distance, the flow is satisfactorily resolved by the direct computation, and the hydrodynamic interactions correctly computed without the need of corrections.

Simulations are performed in a wall-bounded linear shear flow with a domain size $L_{x}=30 \bar{a}$ in velocity direction $(x), L_{y}=40 \bar{a}$ in velocity-gradient $(y)$ direction and $L_{z}=20 \bar{a}$ in vorticity $(z)$ direction. The total number of particles is approximately 4,000 at volume fraction $\phi=0.6$. Upper and lower walls are prescribed with opposite velocities $\pm U_{\text {wall }}$ to produce a linear flow of imposed shear rate $\dot{\gamma}_{i m p}=2 U_{\text {wall }} / L_{y}$. The flow is periodic in velocity $(x)$ and vorticity $(z)$ directions. Computations are conducted during at least $100 \dot{\gamma}^{-1}$ for the lowest volume fractions and typically $20 \sim 30 \dot{\gamma}^{-1}$ for the highest volume fractions. The time step can be as low as $10^{-5} \dot{\gamma}^{-1}$ for the dense frictional cases. In the following results, we consider bulk $\phi, \dot{\gamma}$, and viscosity $\eta$ measured in the core of the suspension to avoid any bias due to a small amount of wall slip or wall layering. 


\section{RESULTS}

\section{A. Three-sphere configuration}

Before moving to many-sphere simulations, we consider a much simpler configuration. The case of three spheres is interesting since it is the simplest case having no analytical solutions. We consider three equally-sized particles (radius $a$ ) in a shear flow and aligned in the direction of compression $(\theta=3 \pi / 4$ oriented from the flow direction), see inset in Fig. 1. This can be thought as the most probable configuration for a triplet in a shear flow. The distance between particles is set to $\xi a$. Both FS and MD simulations are conducted on this configuration for different non-dimensional gap $\xi$ and the relative $L_{1}$-error $E$ is computed between FS and MD for translational particle velocity $E_{u}=\frac{1}{3} \sum\left|\mathbf{u}_{F S}-\mathbf{u}_{M D}\right| /\left|\mathbf{u}_{F S}\right|$ and for particle stresslet $S$ (xy component), i.e. $E_{S}=\frac{1}{3} \sum\left|S_{F S}-S_{M D}\right| /\left|S_{F S}\right|$, where the sum runs over the three particles. Velocities are scaled by $\dot{\gamma} a$ and stresslets by $10 / 3 \pi \dot{\gamma} a^{3}$. Figure 1 presents the obtained errors and carries two main salient points. First, errors on velocity are vanishing for small gaps and is at most $2 \%$ at $\xi=10^{-2}$ meaning that FS and MD give similar outcomes. This confirms that short-range lubrication is the predominant hydrodynamic contribution for small gaps typical of dense suspensions. There is a significant error only for larger gaps (e.g., $\xi=10^{-1}$ ). The discrepancy between FS and MD is larger for the stresslet,

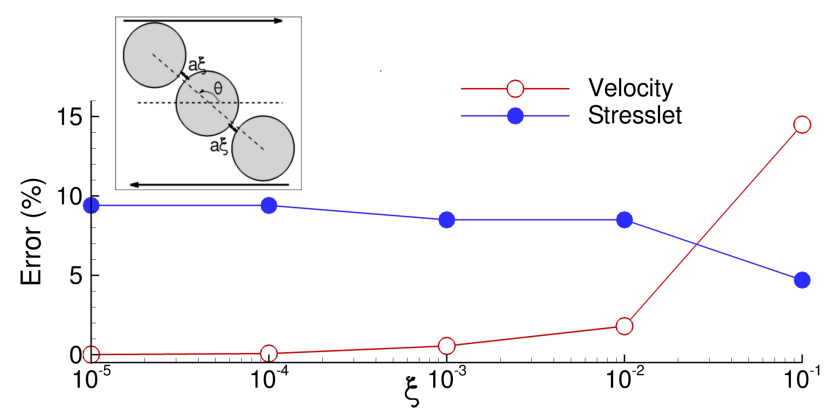

FIG. 1. Error between FS and MD on particle velocity and stresslet against non-dimensional gap distance $\xi$. Inset: sketch of the three-particle configuration.

in the range 5-10\%, even for small gaps. Most of the error actually comes from the two outer particles whose stresslets are slightly underestimated by MD compared to FS. To go further, we have additionally computed this simple configuration by direct simulation, i.e. by solving Stokes equations everywhere in the fluid - even in the lubrication layer between 
particles - using the commercial software Comsol@. In that case, there are no assumptions on lubrication or pairwise approximations. This approach can be conducted on this simple system but would not be tractable for large-scale simulations of many particles because of extremely small grid elements in the lubrication layer between particles. Although not theoretical nor analytical, we will refer this solution as "exact" in the following Table I, which presents results on the computed stresslets for two distances $\xi=10^{-1}$ and $\xi=10^{-3}$. Stresslets for the central and the two outer particles are respectively noted $S_{c}$ and $S_{\text {out }}$ and scaled by $S_{\infty}=10 / 3 \pi a^{3} \dot{\gamma}$. The FS simulations match perfectly well the reference solution, meaning that our lubrication correction strategy is sound and gives accurate predictions. As mentioned previously, MD slightly underestimates stresslets, especially for outer particles.

TABLE I. Computed stresslets for central $\left(S_{c}\right)$ and outer $\left(S_{\text {out }}\right)$ particles in the three-sphere configuration for two gaps.

\begin{tabular}{lccr}
\hline \hline \multirow{3}{*}{$\xi=10^{-3}$} & & $S_{c}$ & $S_{\text {out }}$ \\
\hline & MD & 2.79 & 1.90 \\
& FS & 2.88 & 2.14 \\
& Exact & 2.88 & 2.15 \\
$\xi=10^{-1}$ & MD & & \\
& FS & 2.28 & 1.64 \\
& Exact & 2.19 & 1.73 \\
\hline \hline
\end{tabular}

\section{B. Suspension viscosity}

We now move to an actual many-sphere suspension computed both with MD and FS. The case of frictionless $\left(\mu_{d}=0\right)$ and frictional particles $\left(\mu_{d}=0.5\right)$ is considered. Figure 2 presents the relative viscosity $\eta_{r}$ predicted by FS and MD for the frictionless and frictional case. For MD simulations, we have prescribed $h_{\max } / \bar{a}=0.05$. The results encompass a wide range of volume fraction from $\phi=0.1$ up to $\phi=0.62$ (frictionless) or $\phi=0.56$ (frictional). As a general trend, viscosity curves show very similar results. There are only limited 
discrepancies - especially for the frictionless case at moderate $\phi$ - but for high fractions, MD and FS results are very close. Fundamental studies on dense suspensions generally consider viscosity divergence, so that we have fitted our results against a divergent law of the form $\left(1-\phi / \phi_{m}\right)^{\alpha}$. Solid lines in Fig. 2 are the obtained best fits with parameters provided in Tab. II. It can be seen that friction has a strong impact on jamming fraction $\phi_{m}$ with $\phi_{m} \approx 0.64$ for frictionless and $\phi_{m} \approx 0.58$ for frictional particles. Those values are in good accordance with available numerical $[2,18]$ and experimental results $[19,20]$. A result is that long-range hydrodynamics do not seem to play a strong role close to jamming since jamming fraction $\phi_{m}$ and critical exponent $\alpha$ are relatively unchanged. This confirms a previous numerical work arguing that viscosity divergence weakly depends on the exact dissipation mechanism [5]. Viscosity diverges irrespective of long-range hydrodynamics. In Fig. 2(a), we have also added other MD data taken from Mari et al. [2] in order to confirm our predictions. Note that the models are not exactly similar (e.g., contact repulsion force), which can easily explain the small discrepancies noted, but the agreement is good overall.
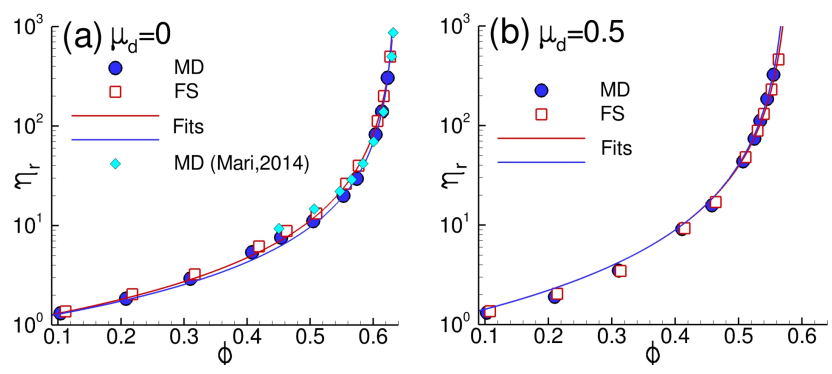

FIG. 2. Predicted relative viscosity for FS and MD against volume fraction for frictionless (a) and frictional (b) particles. Solid lines are a fit against $\left(1-\phi / \phi_{m}\right)^{\alpha}$ (Tab. II). MD simulation results from [2] are also presented in (a).

The relative viscosity $\eta_{r}$ can be split into a solvent contribution, contact contribution, and hydrodynamic contribution: $\eta_{r}=1+\eta_{r}^{c}+\eta_{r}^{h}$. We can therefore study the relative contribution of contacts and hydrodynamics by considering the following ratios $\chi^{h}=\eta_{r}^{h} /\left(\eta_{r}-1\right)$ and $\chi^{c}=\eta_{r}^{c} /\left(\eta_{r}-1\right)$. Such quantities are plotted in Fig. 3 in the frictionless case $\left(\mu_{d}=0\right)$ and Fig. 4 in the frictional case $\left(\mu_{d}=0.5\right)$. If we first consider the frictionless case (Fig. 3), we note that $\chi^{h}$ strongly decreases (and $\chi^{c}$ grows accordingly) for high fractions, showing that viscosity of dense systems is mostly due to contacts. The hydrodynamic contribution $\chi^{h}$ 
TABLE II. Best fit parameters $\left(1-\phi / \phi_{m}\right)^{\alpha}$

\begin{tabular}{lcr}
\hline \hline & $\phi_{m}$ & $\alpha$ \\
\hline FS $\left(\mu_{d}=0\right)$ & 0.640 & -1.59 \\
$\operatorname{MD}\left(\mu_{d}=0\right)$ & 0.636 & -1.47 \\
FS $\left(\mu_{d}=0.5\right)$ & 0.585 & -1.90 \\
$\operatorname{MD}\left(\mu_{d}=0.5\right)$ & 0.581 & -1.88 \\
\hline \hline
\end{tabular}

is slightly lower for MD when volume fraction is low, say $\phi \lesssim 0.45$. Accordingly, in this semi-dilute regime, MD overestimates contacts. The conclusion is similar in the frictional case (Fig. 4) although effects are more amplified by friction. It is much more clear here that MD predicts an underestimated contribution of hydrodynamics (and overestimated role of contacts). By and large, long-range interactions do not strongly modify viscosity but change the repartition between hydrodynamics and contacts ; in particular, a part of the hydrodynamic contribution is missed by MD in semi-dilute regimes especially for frictional particles.

Before we end this section on viscosity, let us mention that we have also investigated the effect of the cut-off distance $h_{\max }$ used in MD simulations. A $h_{\max } / \bar{a}=0.05$ was taken in previous simulations as a typical value from the literature but we have tested other values in the range $0.01-0.2$. For this latter range, the MD viscosity variation is typically within 20-30 \% and viscosity increases with $h_{\max }$. Therefore the reader must keep in mind this dependence of MD predictions to an arbitrary parameter and that agreement is correct because an adequate value of $h_{\max }$ is selected.
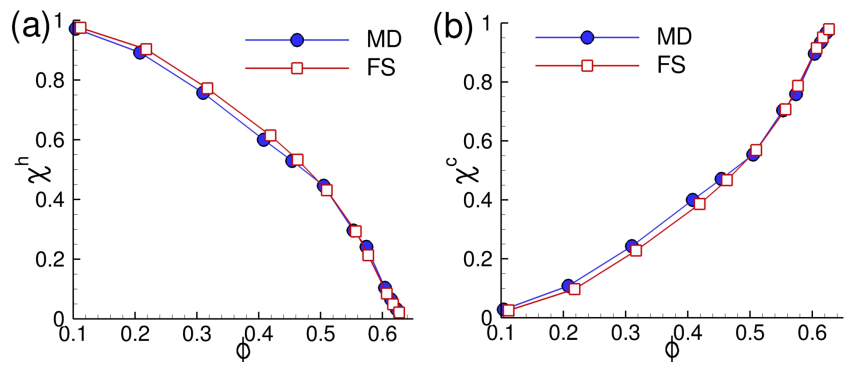

FIG. 3. Relative contribution of hydrodynamics $\chi^{h}$ (a) or contacts $\chi^{c}$ (b) to viscosity: frictionless case. 

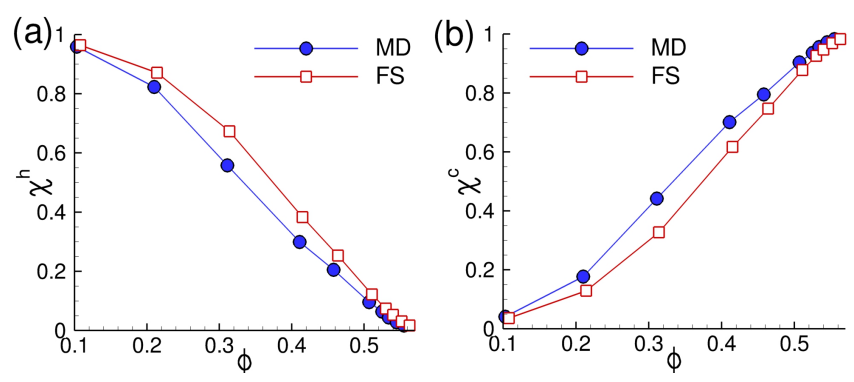

FIG. 4. Relative contribution of hydrodynamics $\chi^{h}$ (a) or contacts $\chi^{c}$ (b) to viscosity: frictional case.

\section{Microstructure and diffusion}

We here investigate the effect of long-range interactions on microstructure and diffusion. Because frictional and frictionless results are qualitatively similar, we here only focus on the frictionless case. Figure 5 presents the pair-correlation function at contact $g_{c}$ plotted as a function of the orientation angle $\theta$ in the shear plane $(x, y)$ for a dilute case $(\phi=0.2)$ and dense case $(\phi=0.6)$. The pair-correlation function $g$ is computed classically in spherical coordinates $g(r, \theta, \psi)$ and $g_{c}$ is such that $g_{c}=g\left(r<r_{c}, \theta, \pi / 2\right)$. We choose $r_{c}=2 a_{2}$ so that all possible contacts are considered. For $\phi=0.2$, the major difference between FS and MD lies in the value of $g_{c}$, which is related to the number of contacts. Long-range interactions lead to a reduced number of actual contacts between particles which explains the lower values of $g_{c}$ in the FS case. It is consistent with our previous result on the smaller contact contribution to viscosity noted in FS simulations. We note however that the angular distribution of contacts remains similar in both cases with a high number of contacting particles in the compression quadrant $\left(0<\theta<90^{\circ}\right)$ and a depletion in the extensional quadrant $\left(90^{\circ}<\theta<180^{\circ}\right)$. For dense conditions $(\phi=0.6)$, there are little differences between FS and MD in $g_{c}$ values or angular distribution. Note that in this case, the microstructure has significantly changed with a high probability of aligned particles $\left(\theta=0^{\circ}\right.$ or $\left.180^{\circ}\right)$.

Diffusion coefficients $D_{y y}$ and $D_{z z}$ in the velocity-gradient and vorticity directions, respectively, are computed in the usual manner by considering the mean-square particle displacements $\langle y y\rangle$ and $\langle z z\rangle$ which grow linearly with time $t$ at long times, i.e. $\langle y y\rangle \sim 2 D_{y y} t$ and $\langle z z\rangle \sim 2 D_{z z} t$. For frictionless particles, the obtained diffusion coefficients (scaled by $\left.\dot{\gamma} \bar{a}^{2}\right)$ are shown in Fig. 6. For all volume fractions, diffusion is predicted to be larger by FS 


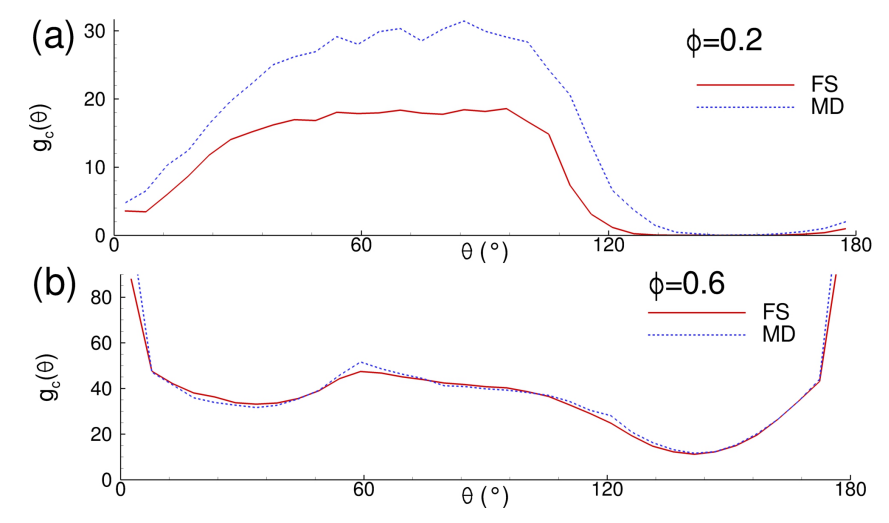

FIG. 5. Pair-correlation $g_{c}$ in the plane of shear as a function of orientation angle $\theta: \phi=0.2$ (a) and $\phi=0.6$ (b). Particles are frictionless.

simulations and the MD underprediction is in the range 1.1-2.3, depending on fraction and direction. Long-range interactions are found to increase diffusion even in dense suspensions although the maximal discrepancy is for dilute regimes (typically a factor 2). Although it is well accepted that diffusion arises from contacts [21], simulations show that long-range hydrodynamics can play a role as well. This role should be included for reliable quantitative predictions. Yet, discarding long-range interactions nevertheless gives an overall qualitatively correct physical picture, confirming that diffusion mostly originates from contacts and is moderately affected by hydrodynamics [21, 22]. In Fig. 6, we note an inflection point, mostly on $D_{y y}$, for $\phi \approx 0.5$, which is thought to be related to a slight tendency for crystallization as attested by the orientational order parameter $Q_{6}$ which is computed to increase in the volume fraction range 0.5-0.57. This incipient order disappears for higher fractions as also noted in [10].
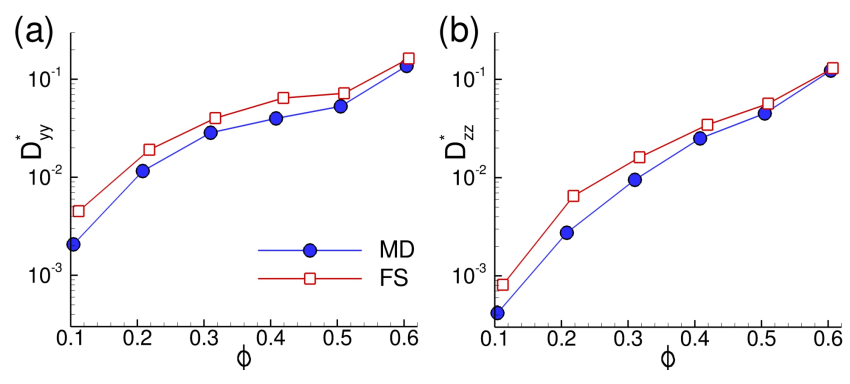

FIG. 6. Scaled diffusion coefficients $D_{y y}^{*}=D_{y y} / \dot{\gamma} \bar{a}^{2}$ (a) and $D_{z z}^{*}=D_{z z} / \dot{\gamma} \bar{a}^{2}$ (b) (frictionless particles).

This effect of long-range interactions can be investigated further by considering particle 
velocity correlations. We have for instance computed the space autocorrelation function $C_{v}$ for particle velocity $v_{p}$ (y-component) in direction $y$ by $C_{v}(y)=\left\langle v_{p}(\mathbf{x}) \cdot v_{p}\left(\mathbf{x}+y \mathbf{e}_{\mathbf{y}}\right)\right\rangle$ where brackets note an average in space and time. This correlation is plotted in Fig. 7 as a function of distance $y$ (scaled by $a_{1}$ ) in the dilute case ( $\phi=0.2$, top panel) and dense case ( $\phi=0.6$, bottom panel). Because the suspension is bidisperse, there are three contact peaks locateddepending on the pair - at $y / a_{1}=2$ (small-small), $y / a_{1}=2 \bar{a} / a_{1}$ (small-large) and $y / a_{1}=2 a_{2} / a_{1}$ (large-large). The results obtained in the dilute case $\phi=0.2$ (Fig. $7(\mathrm{a})$ ) clearly show that velocity is much more correlated when long-range hydrodynamics is included. Conversely, MD simulations show a short-ranged velocity correlation, mostly for pairs and triplets $\left(y / a_{1}\right.$ between 3 and 5.6, depending on particle size and triplet orientation). Above $y / a_{1} \approx 8$, velocity is uncorrelated in MD simulations unlike FS results. This supports that shortrange lubrication interactions can not properly describe the long-range correlated dynamics of suspension in dilute regimes. The contact peaks in Fig. 7(a) are almost invisible in the FS case but quite marked in MD simulations. It indicates that discarding many-body long-range interactions implies that velocity is correlated only for particles in contact. In the dense case $\phi=0.6$ (Fig. 7(b)), the velocity correlation still remains long-ranged in FS simulations and we have $C_{v}(y) \propto y^{-1.6}$ for both volume fractions. Hydrodynamic interactions extend over long distances and are not significantly screened, which agrees with results from Zia et al. [13]. In this dense case, we have a similar behavior between FS and MD simulations which means that pair-interactions through neighboring particles induce an overall longrange behavior. Similarly to diffusion, the way hydrodynamic interactions are handled has a stronger influence in dilute regimes.

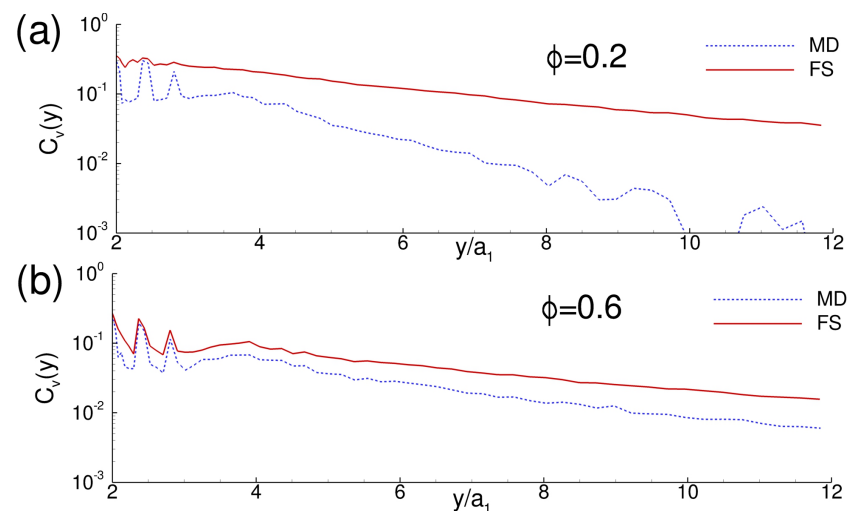

FIG. 7. Velocity correlation $C_{v}(y)$ for $\phi=0.2$ (a) and $\phi=0.6$ (b). Frictionless particles. 


\section{CONCLUSION}

We have performed detailed three-dimensional simulations of sheared non-Brownian, noncolloidal suspensions using two methods with an eye to estimating the role of long-range many-body hydrodynamics. The first approach solves the Stokes equations with contact and short-range lubrication while the second is based on molecular dynamics with only contact and short-range lubrication, thus missing long-range hydrodynamics. Our simulations show that viscosities are similar in both cases, although molecular dynamics overestimates the contribution of contacts. Notably, viscosity diverges at the same critical fraction and with similar exponent. Molecular dynamics underestimates diffusion and misses the long-range nature of particle velocity correlation, except for very concentrated regimes. Long-range hydrodynamics are found not to be significantly screened by crowding. The issue of screening in colloidal suspensions is also of great interest but has not been addressed here because colloidal motion was not included, which could be part of subsequent studies. As a final conclusion, molecular dynamics seems well-suited for rheology but if finer flow details (e.g., velocity correlation, diffusion) are needed, it might be more suitable to consider Stokes flow simulations, even in concentrated suspensions. Since shear-induced migration is connected to diffusion [23], it is expected that the way hydrodynamic interactions are handled in simulations is likely to have significant effects on the prediction of migration.

[1] J.J. Stickel and R.L. Powell. Fluid mechanics and rheology of dense suspensions. Annu. Rev. Fluid Mech., 37:129-149, 2005.

[2] R. Mari, R. Seto, J.F. Morris, and M.M. Denn. Shear thickening, frictionless and frictional rheologies in non-brownian suspensions. J. Rheol., 58(6):1693-1724, 2014.

[3] M. Trulsson, B. Andreotti, and P. Claudin. Transition from the viscous to inertial regime in dense suspensions. Phys. Rev. Lett., 109(11):118305, 2012.

[4] C. Ness and J. Sun. Flow regime transitions in dense non-brownian suspensions: Rheology, microstructural characterization, and constitutive modeling. Phys. Rev. E, 91(1):012201, 2015.

[5] B. Andreotti, J.L. Barrat, and C. Heussinger. Shear flow of non-brownian suspensions close to jamming. Phys. Rev. Lett., 109(10):105901, 2012. 
[6] R.C. Ball and J.R. Melrose. A simulation technique for many spheres in quasi-static motion under frame-invariant pair drag and brownian forces. Physica A, 247(1-4):444-472, 1997.

[7] J.F. Brady and G. Bossis. The rheology of concentrated suspensions of spheres in simple shear flow by numerical simulation. J. Fluid Mech., 155:105-129, 1985.

[8] K. Yeo and M.R. Maxey. Dynamics of concentrated suspensions of non-colloidal particles in couette flow. J. Fluid Mech., 649:205-231, 2010.

[9] S. Gallier, E. Lemaire, F. Peters, and L. Lobry. Rheology of sheared suspensions of rough frictional particles. J. Fluid Mech., 757:514-549, 2014.

[10] A. Sierou and J.F. Brady. Rheology and microstructure in concentrated noncolloidal suspensions. J. Rheol., 46(5):1031-1056, 2002.

[11] Z. Varga, G. Wang, and J. Swan. The hydrodynamics of colloidal gelation. Soft Matter, 11(46):9009-9019, 2015.

[12] X.J. Cao, H.Z. Cummins, and J.F. Morris. Hydrodynamic and interparticle potential effects on aggregation of colloidal particles. J. Colloid Interface Sci., 368(1):86-96, 2012.

[13] R.N. Zia, J.W. Swan, and Y. Su. Pair mobility functions for rigid spheres in concentrated colloidal dispersions: Force, torque, translation, and rotation. J. Chem. Phys., 143(22):224901, 2015.

[14] A. Shauly, A. Wachs, and A. Nir. Shear-induced particle migration in a polydisperse concentrated suspension. J. Rheol., 42(6):1329-1348, 1998.

[15] S. Gallier, E. Lemaire, L. Lobry, and F. Peters. A fictitious domain approach for the simulation of dense suspensions. J. Comp. Phys., 256:367-387, 2014.

[16] A. Kumar. Microscale dynamics in suspensions of non-spherical particles. PhD thesis, 2010.

[17] J.R. Smart and D.T. Leighton. Measurement of the hydrodynamic surface roughness of noncolloidal spheres. Phys. Fluids, 1(1):52-60, 1989.

[18] F. Peters, G. Ghigliotti, S. Gallier, F. Blanc, E. Lemaire, and L. Lobry. Rheology of nonbrownian suspensions of rough frictional particles under shear reversal: A numerical study. $J$. Rheol., 60(4):715-732, 2016.

[19] G. Ovarlez, F. Bertrand, and S. Rodts. Local determination of the constitutive law of a dense suspension of noncolloidal particles through magnetic resonance imaging. J. Rheol., 50(3):259-292, 2006. 
[20] F. Boyer, E. Guazzelli, and O. Pouliquen. Unifying suspension and granular rheology. Phys. Rev. Lett., 107(18):188301, 2011.

[21] P. Pham, B. Metzger, and J.E. Butler. Particle dispersion in sheared suspensions: Crucial role of solid-solid contacts. Phys. Fluids, 27(5):051701, 2015.

$[22]$ B. Metzger, and J.E. Butler. Irreversibility and chaos: Role of long-range hydrodynamic interactions in sheared suspensions. Phys. Rev. E, 82(5):051406, 2010.

[23] A.M. Leshansky and J.F. Brady. Dynamic structure factor study of diffusion in strongly sheared suspensions. J. Fluid Mech., 527:141-169, 2005. 